\title{
Schwannoma on the nasal dorsum and tip with sensory changes
}

\author{
Sung Ho Yoon, \\ Cha Soo Kim, \\ Jae Wook Oh, \\ Keun Cheol Lee \\ Department of Plastic and \\ Reconstructive Surgery, Inje University \\ Haeundae Paik Hospital, Inje University \\ College of Medicine, Busan, Korea
}

Schwannomas, which originate from Schwann cells in the peripheral nervous system, are slowgrowing and uncommon benign tumors. Most schwannomas (90\%) occur in isolation, and multiple occurrences are a characteristic feature of neurofibromas. Schwannomas of the nose and nasal tip are particularly unusual. Although a few cases of schwannomas of the sinusoidal tract and nasal septum have been reported, schwannomas arising from the nasal dorsum area and tip are extremely rare. Sensory abnormalities are also a very rare symptom. We excised a schwannoma on the nasal dorsum through direct incision and a schwannoma on the nasal tip through open rhinoplasty. No postoperative complications involving recurrence, hematoma, or infection occurred. The possibility of neurological changes should be considered in cases of an abnormality in the peripheral nervous system. Schwannoma must be kept in mind as a possible cause of neurological changes localized to a specific dermatome, and should always be considered in the differential diagnosis of a mass on the nose.

Keywords: Neuroma / Nose neoplasm / Sensation

\section{INTRODUCTION}

Schwannomas and neurofibromas are the most common types of peripheral nerve sheath tumors. Schwannomas, which originate from Schwann cells in the peripheral nervous system, are slow-growing and uncommon benign tumors. Approximately $25 \%$ to $45 \%$ of schwannomas that occur outside of the cranial cavity are located in the head and neck [1]. Roughly $4 \%$ of schwannomas arise from the sinonasal tract, and schwannomas of the nose and nose tip are particularly unusual [2-4]. Although a few cases of schwannomas of the sinusoidal tract and nasal septum have been reported, schwannomas arising from the nasal dorsum area and tip are extremely rare [2-4]. Most symptoms caused by schwannomas in the nose are caused by

Correspondence: Keun Cheol Lee

Department of Plastic and Reconstructive Surgery, Inje University Haeundae Paik Hospital, Inje University School of Medicine, 875 Haeun-daero, Haeundae-gu,

Busan 48108, Korea

E-mail: H00511@paik.ac.kr

Received August 10, 2020 / Revised October 13, 2020 / Accepted December 8, 2020 mass effects [1,5-8]. However, in the two patients described herein, the symptoms intensified as the mass grew, with hypoesthesia in the dermatomes innervated by certain nerves. The symptoms improved postoperatively, but sensation did not recover completely. In these two cases of schwannomas of the nasal dorsum and tip, surgical resection resulted in functionally and cosmetically successful outcomes.

\section{CASE REPORTS}

\section{Case 1}

A 12-year-old girl visited the plastic surgery department with a chief complaint of a growing mass on the dorsum of her nose. She had no history of nasal trauma, underlying disease, or congenital disease. The mass measured approximately $2.3 \times 2.5 \mathrm{~cm}$, had gradually grown over the past month, and was round and movable. The patient did not present with any noteworthy symptoms, including skin color changes or blockage of the nasal cavity, and the size of the nasal cavity on both sides was 
identical. She reported no discomfort other than slight pain associated with the growth of the mass and mild hypoesthesia on the nasal dorsum. We conducted two-point discrimination testing using a caliper to assess sensory impairment, and the result was $15-17 \mathrm{~mm}$ (reference: 10-12 $\mathrm{mm}$ ) [9]. The patient complained of sensory abnormalities precisely in the area innervated by the infratrochlear nerve, leading to the suspicion of schwannoma in this area. Preoperative ultrasonography revealed a heterogeneous, echoic, solid cystic mass that did not invade the nasal septum, cartilage, mucosa, or bone. T2 magnetic resonance imaging (MRI) showed a round, well-defined, hyperintense mass. A direct incision was made because the mass was large; therefore, the open rhinoplasty approach would have been limited to the adhesion between the mass and soft tissue and securing the field of view. Under general anesthesia, an incision was made directly over the mass and resection was performed successfully. The round, encapsulated mass was completely removed. The patient had no significant complications and reported high cosmetic satisfaction. The diagnosis was confirmed by a definitive histopathological examination.
The patient was monitored for 7 months after surgery, and an appropriate nasal contour was maintained. At a follow-up visit, we performed two-point discrimination testing around the mass. Sensation had improved relative to the preoperative findings, but had not returned to normal. There were no signs of recurrence or complications (Fig. 1).

\section{Case 2}

A 38-year-old man with no underlying disease presented to the plastic surgery department with a chief complaint of a growing mass at the center of his nose. The mass was roughly $2.3 \times 1.8$ $\mathrm{cm}$. The patient had first noticed the mass approximately 6 months ago, but had not previously sought treatment. He complained of slight nasal congestion and numbness, but reported no other specific symptoms. We conducted two-point discrimination testing to assess sensory impairment, and the result was 15-17 mm (reference: 10-12 mm) [9]. The patient complained of impaired sensation at the tip of the nose and in the nose, corresponding to areas innervated by the nasociliary nerve. The mass was removed under local anesthesia using an open ap-
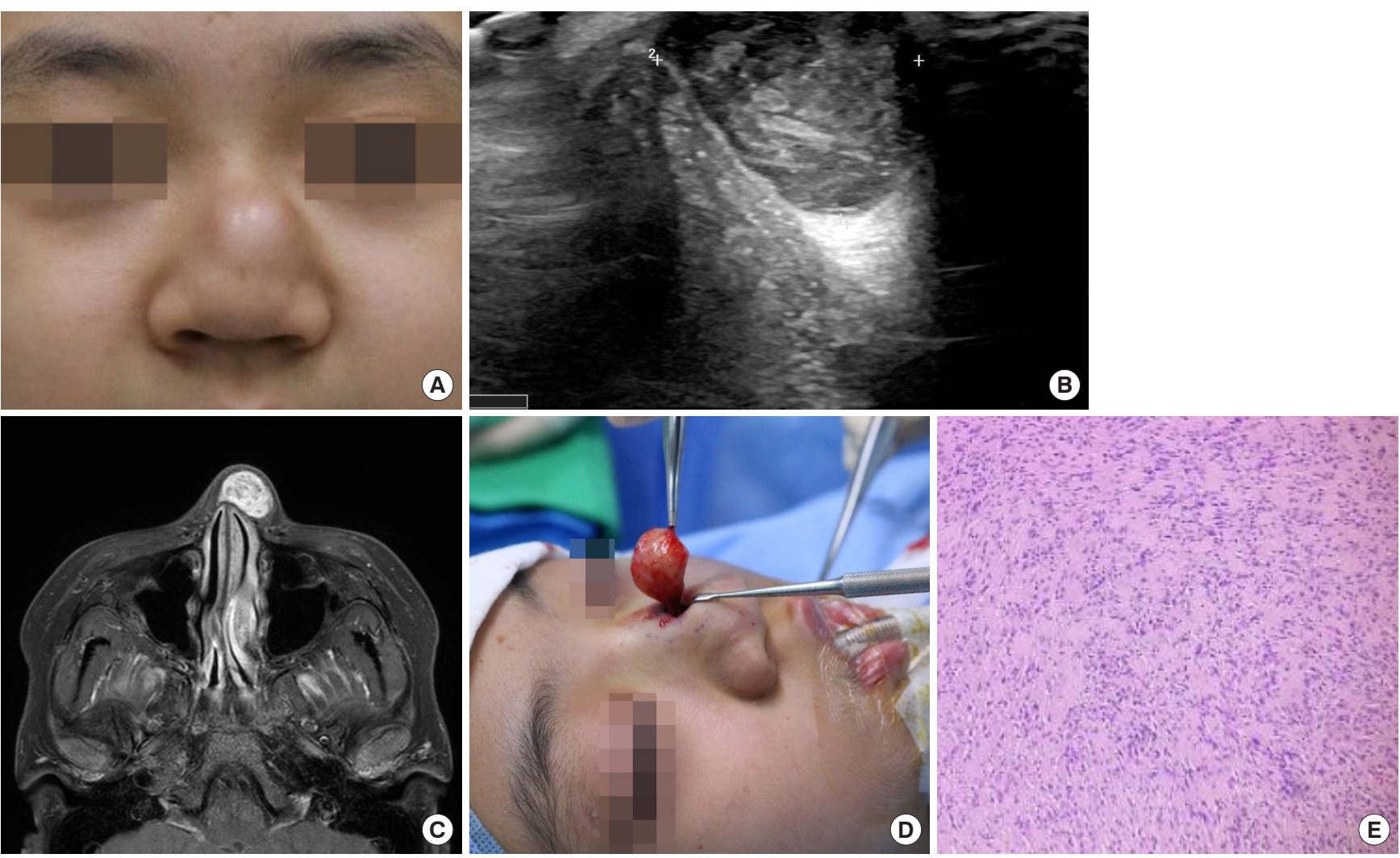

Fig. 1. A 12-year-old girl case of a growing mass on the nasal dorsum with hypoesthesia. (A) Preoperative photograph of case 1. (B) Sonographic image showing a heterogeneous, echoic, solid cystic mass. (C) Axial view of a T2-weighted magnetic resonance image, showing a well-circumscribed, cystic mass on the nasal dorsum. The mass showed a homogeneously high signal and was separated from the soft tissue. (D) Intraoperative photograph of the mass, which was totally resected with the capsule. (E) A histological section showed the tumor to be composed of spindleshaped cells with regular nuclei, without mitotic activity, but with some tendency for palisade formation (H\&E, $\times 100)$. 

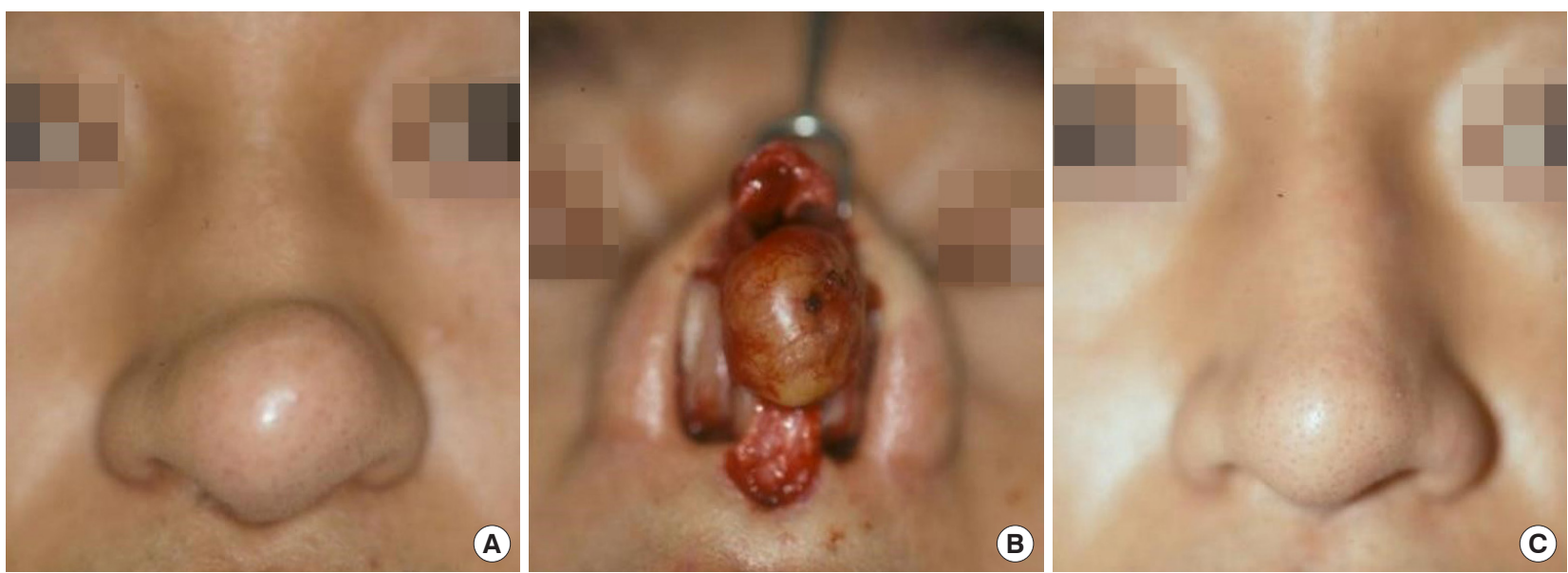

Fig. 2. A 38-year-old man case showing a mass on the nasal tip. (A) Preoperative photograph of case 2. (B) Intraoperative photograph of the mass, which was totally resected with the capsule. (C) Postoperative photograph.

proach, and rhinoplasty with cartilage repositioning was performed. As with the first patient, sensation did not fully recover, but impaired sensation did not interfere with the patient's daily life. During 1 year of postoperative follow-up, neither recurrence nor complications were observed (Fig. 2).

\section{DISCUSSION}

A schwannoma is a benign tumor originating from the nerve sheath [1]. It is a nodular or spindle-shaped, elongated mass formed by proliferation of the Schwann cells in the nerve sheath of the peripheral, cranial, and sympathetic nerves [1]. Schwannomas are encapsulated, unaccompanied by axonal proliferation, and confined within the endoneurium, thereby causing no damage to the epineurium. Most schwannomas (90\%) occur in isolation, whereas multiple occurrences are a characteristic feature of neurofibromas. Neurofibromas have a higher likelihood of becoming malignant than do schwannomas [10]. Malignant schwannomas are very rare, but can be suspected when the following diagnostic criteria are met: metastasis to other organs, presence of nuclear atypia or mitotic activity, or rapid deterioration of the patient's systemic condition, as shown by symptoms such as weight loss. The clinical manifestations of schwannomas can result from direct involvement of the nerve from which the tumor arose, but mostly from mechanical compression of nearby nerves by the tumor [11]. Involvement of a sensory or motor nerve may result in symptoms associated with the function of the specific nerve, such as facial paralysis, hearing loss, dyspnea, and cough; however, if the trigeminal nerve is involved, it is usually asymptomatic. However, despite being derived from nerve tissues, a schwannoma does not cause pain unless pressure is applied to the surrounding nerves. Overall, the most common symptom is discomfort caused by the mass [12].

Schwannoma should be differentiated from other tumors of the nose, including nasal polyp, pleomorphic adenoma, angiofibroma, dermoid cyst, and inverted papilloma [10]. Due to the wide variety of possible diagnoses, clinical findings are not sufficient for the differential diagnosis in most cases. Although schwannomas do not have distinct imaging characteristics on computed tomography (CT) and MRI, these imaging modalities can be useful for identifying the size and extent of a lesion before surgical resection. Furthermore, CT and MRI findings can provide guidance for the initial differential diagnosis [7]. For example, if there is no sinus tract or brain herniation, intranasal glioma or encephalocele can be excluded. In adolescent patients, juvenile angiofibroma or congenital disease should be suspected, and nasal bleeding and contrast enhancement may suggest a vascular tumor [8]. Evidence of bone remodeling and slow growth of a lesion suggest an increased likelihood of benignity, whereas bone destruction is a useful indicator of an inverted papilloma or other malignancy [8]. Therefore, imaging tests allow a rough prediction of whether a lesion of the nose is malignant.

Surgical resection is the treatment of choice for most schwannomas [13]. As they are well encapsulated, complete removal is relatively easy; however, care should be taken to avoid causing damage to the sensory and motor nerves when important nerves are involved, and incomplete resection can lead to local recurrence. Recurrence of schwannomas after complete removal is rare $[1,3,4]$.

In case 1 , the mass was surgically removed through a direct incision. In case 2 , the mass was resected via open rhinoplasty (the step-ladder pattern) and no recurrence or complications were observed during the follow-up period. 
Most schwannomas do not cause symptoms [1,3-8,14]. When neurological symptoms do occur, they are almost always due to the mass effect, which occurs as the mass grows and compresses nerves. However, in the two patients described herein, neurological symptoms preceded the diagnosis of the mass. As the mass became larger, the neurological symptoms also became more severe. In other words, schwannoma should be suspected in patients with neurological symptoms.

Schwannomas may develop anywhere in the body and arise from the myelin sheaths of peripheral motor, sensory, sympathetic, and cranial nerves [1]. Schwannomas of the nose with sensory changes are extremely rare. Our experience of successful treatment with surgical resection suggests that schwannoma should be considered in the differential diagnosis of masses located at the nasal dorsum, as well as those at the nasal tip.

\section{NOTES}

\section{Conflict of interest}

No potential conflict of interest relevant to this article was reported.

\section{Ethical approval}

The study was approved by the Institutional Review Board of Inje University Haeundae Paik Hospital (IRB No. 2020-07-038) and performed in accordance with the principles of the Declaration of Helsinki. Written informed consent was obtained.

\section{Patient consent}

The patients provided written informed consent for the publication and the use of their images.

\section{ORCID}

Sung Ho Yoon

Cha Soo Kim

Jae Wook Oh

Keun Cheol Lee https://orcid.org/0000-0003-4765-2308 https://orcid.org/0000-0002-2451-3025 https://orcid.org/0000-0001-8829-0935 https://orcid.org/0000-0003-0553-0900

\section{REFERENCES}

1. Colreavy MP, Lacy PD, Hughes J, Bouchier-Hayes D, Brennan P, O'Dwyer AJ, et al. Head and neck schwannomas: a 10 year review. J Laryngol Otol 2000;114:119-24.

2. Dublin AB, Dedo HH, Bridger WH. Intranasal schwannoma: magnetic resonance and computed tomography appearance. Am J Otolaryngol 1995;16:251-4.

3. Gul E, Piechnik-Resler D, Gul A. A case of schwannoma of the membranic part of nasal septum. Otolaryngol Pol 2005;59:84952.

4. Donnelly MJ, al-Sader MH, Blayney AW. Benign nasal schwannoma. J Laryngol Otol 1992;106:1011-5.

5. Mohan D, Krishna A, Ramakristnan V. Schwannoma presenting as a nasal tip deformity. Ann Plast Surg 1997;38:83-4.

6. Berlucchi M, Piazza C, Blanzuoli L, Battaglia G, Nicolai P. Schwannoma of the nasal septum: a case report with review of the literature. Eur Arch Otorhinolaryngol 2000;257:402-5.

7. Lemmerling M, Moerman M, Govaere F, Praet M, Kunnen M, Vermeersch H. Schwannoma of the tip of the nose: MRI. Neuroradiology 1998;40:264-6.

8. Lee EJ, Kim MK, Kang MS, Kim KS. Nasal tip schwannoma coexisting with vestibular schwannoma. J Craniofac Surg 2016; 27:e626-7.

9. Siemionow M, Gharb BB, Rampazzo A. The face as a sensory organ. Plast Reconstr Surg 2011;127:652-62.

10. Min HJ, Kim KS. Differential diagnosis between nasal septal schwannoma and nasal septal neurofibroma. J Craniofac Surg 2017;28:1780-3

11. Choi JS, Park SM, Choi KU. A rare case of isolated schwannoma in infraorbital nerve. Arch Craniofac Surg 2018;19:231-4.

12. Guthikonda B, Theodosopoulos PV, van Loveren H, Tew JM Jr, Pensak ML. Evolution in the assessment and management of trigeminal schwannoma. Laryngoscope 2008;118:195-203.

13. Min HJ, Hong SC, Kim KS. Nasal septal schwannoma: advances in diagnosis and treatment. J Craniofac Surg 2017;28:e97101.

14. Kim KS, Jung JW, Yoon KC, Kwon YJ, Hwang JH, Lee SY. Schwannoma of the orbit. Arch Craniofac Surg 2015;16:67-72. 